\title{
Synthesis of Green/Blue Light Emitting Quinolines by Aza-D-A Reaction Using $\mathrm{InCl}_{3}$ Catalyst
}

\author{
Rajkumar Romeshkumar Singh ${ }^{1}$. Thokchom Prasanta Singh ${ }^{1}$ • Ningthoujam Premananda Singh ${ }^{1}$. \\ Shanta Singh Naorem ${ }^{2} \cdot$ Okram Mukherjee Singh ${ }^{1}$
}

Received: 8 October 2020 / Accepted: 30 October 2020 / Published online: 20 November 2020

(C) Springer Science+Business Media, LLC, part of Springer Nature 2020

\begin{abstract}
An efficient $\mathrm{InCl}_{3}$-catalyzed sequential reaction of aromatic amines, aromatic aldehydes and functionalized alkynes leading to the formation of new quinoline derivatives exhibiting significant fluorescence activities is described. The photophysical investigations of quinolines were carried out by absorption and photoluminescence measurements. One particular compound $4 \mathbf{h}$ having maximum intensity, emitting green colour $(\Phi=0.78)$ with average life time of $6.20 \mathrm{~ns}$ was the best amongst the tested compounds. The presence of the amino group at the 4-aryl substituent of the quinoline backbone played an important role in executing the Povarov cyclization successfully and enhancing the flourescence properties of the newly synthesized quinolines.
\end{abstract}

Keywords 2-Ethynylaniline $\cdot$ Quinolines $\cdot$ Life time $\cdot$ Photophysical properties $\cdot$ Povarov reaction

\section{Introduction}

Quinolines constitute an important class of $N$-based heterocyclic aromatic compounds occurred as natural products and synthetic complex organic molecules [1-4]. They are well known for exhibiting broad spectrum of biological activities like antitumor, antimalarial, antibacterial, antifungal, antiparasitic and insecticidal, antiviral, anti-inflammatory, antiplatelet and other activities [5-8]. The most well-known and significant quinoline alkaloids are chloroquinine and hydroxychloroquine as antimalarial drugs recently associated with the treatment of the pandemic SARS-CoV-2 [9] and camptothecin an anticancer drug $[10,11]$ development respectively, (Fig. 1). In addition to bioactivity, quinolines scaffolds also show luminescent properties with potential applications in organic solar cells (OSCs), organic light emitting diodes (OLEDs), biomolecular markers, molecular probes and

Rajkumar Romeshkumar Singh and Thokchom Prasanta Singh contributed equally to this work.

Okram Mukherjee Singh

ok_mukherjee@yahoo.co.in

1 Chemistry Department, Manipur University, Canchipur 795003, India

2 Chemistry Department, Nagaland University, Lumami 798627, India switches [12-14]. Moreover, quinoline-based dyes such as ethyl red iodide and pinacyanol (Fig. 1) have been used since the beginning of the nineteenth century in photographic plates [15] The diverse applications of quinolines as functional materials is related to its excellent mechanical properties and high quantum yields, making ideal materials in the electron transport [16] and presenting essential characteristics for their subsequent use in OLEDs [17]. Hence, a significant advance in luminescence efficiency and brightness in OLEDs is observed when conjugated organic compounds contain quinoline moeties [18] Povarov reaction (aza Diel-Alder reaction) [19] remains one of the most efficient methods affording highly substituted and densely functionalized quinoline frameworks. Povarov reaction involves $[4+2]$ cycloaddition reaction of $N$ aryl imines with electron-rich dienophiles via activation of a terminal alkyne $\mathrm{C}-\mathrm{H}$ bond and complexation of $\mathrm{C}-\mathrm{C}$ multiple bonds to facilitate $\mathrm{C}-\mathrm{N}$ and $\mathrm{C}-\mathrm{C}$ bond formation, which are key intermediates for the construction of quinolines [20]. Recently, many metal salts like $\mathrm{CuCl} / \mathrm{AuCl}$ [21] $\mathrm{AuCl}_{3}$ / $\mathrm{CuBr}$ [22], $\mathrm{Yb}(\mathrm{OTf})_{3}$ [23], $\mathrm{Fe}(\mathrm{OTf})_{3}$ [24], $\mathrm{Cu}(\mathrm{OTf})_{2}$ [25], $\mathrm{Zn}(\mathrm{OTf})_{2}$ [26], AgNTf [27], $\mathrm{NbCl}_{5}$ [28] are explored as effective Lewis acid catalytic system for quinoline synthesis though Povarov started with $\mathrm{BF}_{3} \cdot \mathrm{OEt}_{2}$ in his original work [19]. Thus, the development of simple and efficient protocols for quinolines containing unique substituent from readily available starting materials is of great interest to organic chemists. 
Fig. 1 Biological and fluorescent actives Quinolines<smiles>[R]CCN(CC)CCCC(C)Nc1ccnc2cc(Cl)ccc12</smiles><smiles>CC[C@@]1(O)C(=O)OCc2c1cc1n(c2=O)Cc2cc3ccccc3nc2-1</smiles>

Camptothecin<smiles>CCN1C=C/C(=C\c2ccc3ccccc3[n+]2CC)c2ccccc21</smiles>

Ethyl red iodide<smiles>CCN1C(=CC=Cc2ccc3ccccc3[n+]2CC)C=Cc2ccccc21</smiles>

Pinacyanol
Indium trichloride $\left(\mathrm{InCl}_{3}\right)$ has been widely used in organic transformations for the construction of complex heterocycles [29-31]. In continuation of our efforts on exploring the catalytic potential of $\mathrm{InCl}_{3}$ for the synthesis of novel $N$-heterocycles [32-34], we herein report the $\mathrm{InCl}_{3}$-mediated efficient synthesis and the optical characterization of new 2,4-disubstituted quinoline derivatives having potential application as dyes in organic electronic devices (Scheme 1).

\section{Materials and Methods}

\section{Reagents and Instruments}

All reagents and solvents were obtained from commercial suppliers and used without further purification. All reagents were weighed and handled in air at room temperature. For compounds (4a-i) ${ }^{1} \mathrm{H}$ NMR $(400 \mathrm{MHz})$ and ${ }^{13} \mathrm{C}$ NMR $(100 \mathrm{MHz})$ spectra were recorded on Bruker spectrometer using $\mathrm{CDCl}_{3}$ whereas ${ }^{1} \mathrm{H}$ NMR (400 MHz) spectrum for compound $4 \mathrm{~b}$ was recorded on FT-NMR spectrometer using $\mathrm{CDCl}_{3}$. Chemical shifts $\delta$ are in parts per million (ppm) with
$\mathrm{CDCl}_{3}$ as solvent and are relative to tetramethylsilane (TMS) as the internal reference. Data are reported as follows: chemical shift, multiplicity $(\mathrm{s}=$ singlet, $\mathrm{d}=$ doublet, $\mathrm{dd}=$ double doublet, $\mathrm{t}=$ triplet, $\mathrm{m}=$ multiplet) and coupling constants $(J)$ in Hertz. The FT-IR spectra were recorded on a FT-IR spectrometer $(\mathrm{KBr})$. Gas chromatography-electron impact mass spectrometry (GC-EIMS) spectra were measured on a Varian spectrometer using ionization by fast atom bombardment (FAB). Melting points were determined on a "Veego" capillary melting point apparatus and are uncorrected. All the luminescence spectra and quantum yield were recorded using Horiba Fluoromax 4 Spectrophotometer. Samples were dissolved in different solvents and $2 \mathrm{~mL}$ of each solution was put in a $3 \mathrm{~mL}$ quartz cuvette and it was mounted on the sample holder. All the measurements were carried out at room temperature. For quantum yield measurement, the above cuvette containing sample solutions was put inside an integrated sphere and the measurement of both emission and excitations were recorded in the form of emission spectra only.

For the lifetime measurements, time-correlated single photon count (TCSPC) technique was used with the help of Horiba DeltaFlex instrument. $2 \mathrm{~mL}$ of solution which contain
Scheme 1 Quinoline synthesis by Povarov reaction using $\mathrm{InCl}_{3}$

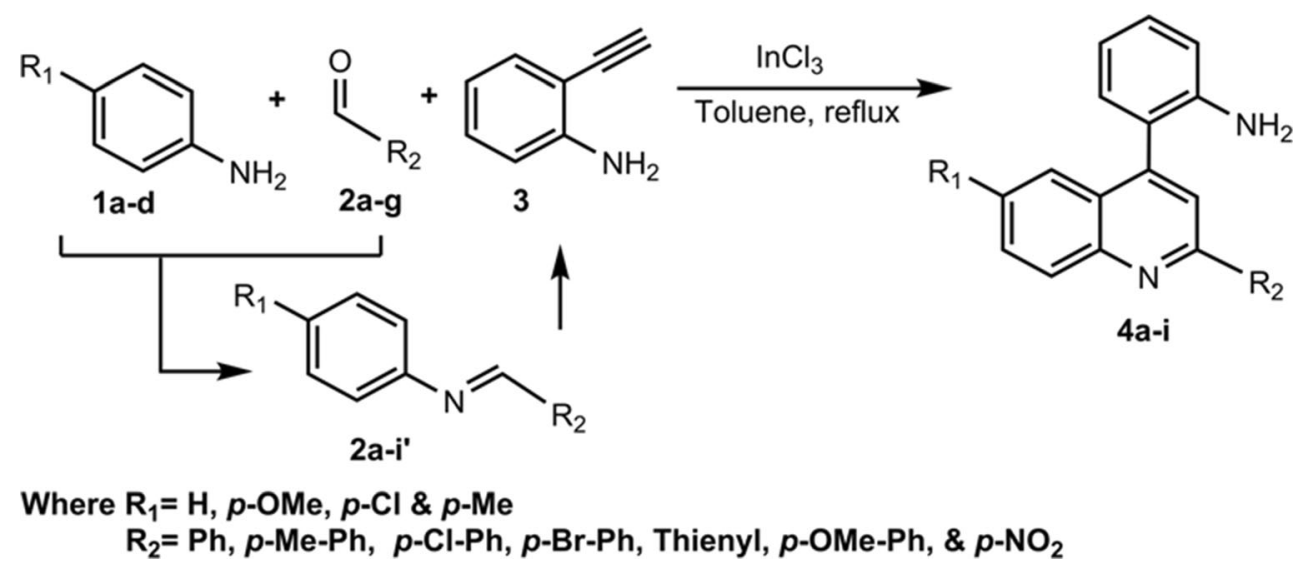


sample was put in a quartz cuvette and it was mounted on the sample holder.

\section{General Procedure for the Preparation of 4a-f}

An equimolar mixture of aromatic amines $1(1.0 \mathrm{mmol})$ and aromatic aldehydes $2(1.0 \mathrm{mmol})$ in presence of $10 \mathrm{~mol} \%$ of $\mathrm{InCl}_{3}$ were refluxed in $5 \mathrm{~mL}$ of toluene. Then after refluxing for $1 \mathrm{~h}$ (as indicated by TLC), 2-ethynylaniline 3 (1.0 $\mathrm{mmol}$ ) was added to reaction pot and refluxed the system for another $23 \mathrm{~h}$. Then, the completions of the reactions were monitored by UV-lamp (giving distinct blue/green colouration). The reaction mixture was brought to room temperature and extracted with ethylacetate $(2 \times 10 \mathrm{~mL})$. The organic layer containing the quinoline was dried over anhydrous $\mathrm{Na}_{2} \mathrm{SO}_{4}$ and then evaporated. The crude residue was purified by column chromatography over silica gel using ethyl acetate/petroleum ether (1:5) as eluent to afford pure product $\mathbf{4 a - i}$ in good yields.

\section{Characterization Data of the Isolated Compounds 4a-I}

1. 2-(2-Phenylquinolin-4-Y1)Aniline (4a): The crude substance purified by gravity column chromatography(ethyl acetate/n-hexane 1:2).

White solid, yield $0.21 \mathrm{~g}(70 \%)$, m.p. $173-175^{\circ} \mathrm{C}$; $\mathrm{R}_{\mathrm{f}}: 0.70$ $\left(\mathrm{SiO}_{2}\right.$, ethyl acetate/n-hexane 1:2), FTIR V: 3389, 3020(N-H); 2929, 2854(aromatic $=$ C-H stretching); 1903, 1614(aromatic = C-H bending), 1519 $(\mathrm{C}=\mathrm{C}) ; 1093,806$ (mono-substituted ring $\mathrm{C}-\mathrm{H}) \mathrm{cm}^{-1},{ }^{1} \mathrm{H}$ NMR (400 $\left.\mathrm{MHz}, \mathrm{CHCl}_{3}-d\right): \delta$ (ppm): 3.59 (s, 2H, $\left.\mathrm{NH}_{2}\right), 6.93-6.85$ (m, 3H. Ar. $\left.=\mathrm{CH}\right), 7.04-7.00$ $(\mathrm{m}, 2 \mathrm{H}, \mathrm{Ar} .=\mathrm{CH}), 7.21-7.19(\mathrm{~m}, 1 \mathrm{H}, \mathrm{Ar} .=\mathrm{CH}), 7.0-7.04(\mathrm{~d}$, $2 \mathrm{H}, \mathrm{Ar} .=\mathrm{CH}, J: 6.4 \mathrm{~Hz}), 8.12$ (d, 3H, J: $7.2 \mathrm{~Hz}),{ }^{13} \mathrm{C} \mathrm{NMR}$ $\left(100 \mathrm{MHz}, \mathrm{CHCl}_{3}-d\right) \delta$ (ppm): 103.7 (Ar. CH), 114.2 (Ar, $\mathrm{CH}$ ), 115.7 (Ar, CH), 118.5 (Ar. CH), 120.0 (Ar. CH), 122.1(Ar. CH), 123.6 (Ar. CH), 126.4(Ar. CH), 128.6 (Ar. $\mathrm{CH}$ ), 129.6 (Ar. $\mathrm{CH}$ ), 130.6 (Ar. $\mathrm{CH}$ ), 131.4 (Ar. $\mathrm{CH}$ ), 132.2 (Ar. CH), 138.8 (Ar. CH), 144.8 (Ar. CH), 145.1 (Ar. $\mathrm{CH}), 154.6$ (Ar. CH), 157.8 (Ar. CH), ESI-MS: $[\mathrm{M}+\mathrm{H}]^{+} \mathrm{m} / \mathrm{z}$ : 297; Chemical Formula: $\mathrm{C}_{21} \mathrm{H}_{16} \mathrm{~N}_{2}$.

2. 2-(6-Methoxy-2-(p-Tolyl)Quinolin-4-Y1)Aniline (4b): The crude substance purified by gravity column chromatography(ethyl acetate/n-hexane 1:2).

White solid, yield 0.24 g (73\%), m.p. $156-158{ }^{\circ} \mathrm{C}$; $\mathrm{R}_{\mathrm{f}}: 0.70$ $\left(\mathrm{SiO}_{2}\right.$, ethyl acetate/n-hexane 1:2), FTIR V: 3375, 3025(N-H), 2909, $2867($ aromatic $=\mathrm{C}-\mathrm{H}), 1903,1617($ aromatic $=\mathrm{C}-\mathrm{H})$, $1511\left(\mathrm{C}=\mathrm{C}\right.$ ), 1097, 810 (mono-substituted ring $\mathrm{C}-\mathrm{H}$ ), ${ }^{1} \mathrm{H}$ NMR (400 MHz, $\left.\mathrm{CHCl}_{3}-d\right): \delta(\mathrm{ppm}): 2.42\left(\mathrm{~s}, 3 \mathrm{H},=\mathrm{CH}_{3}\right.$ ), $3.78\left(\mathrm{~s}, 3 \mathrm{H},=\mathrm{OCH}_{3}\right), 6.95-6.85(\mathrm{~m}, 3 \mathrm{H}, \mathrm{Ar} .=\mathrm{CH}), 7.21(\mathrm{~d}, 1 \mathrm{H}$, Ar. $=\mathrm{CH}, J: 7.6 \mathrm{~Hz}), 7.30$ (t, Ar. $=\mathrm{CH}, J: 8.0 \mathrm{~Hz}), 7.40-7.37(\mathrm{~m}$, $2 \mathrm{H}, \mathrm{Ar} .=\mathrm{CH}), 7.82(\mathrm{~s}, 1 \mathrm{H}, \mathrm{AR} .=\mathrm{CH}), 8.14-8.04(\mathrm{dd}, 3 \mathrm{H}, \mathrm{Ar} .=$
$\mathrm{CH}, \mathrm{J}: 9.2,8.0 \mathrm{~Hz}),{ }^{13} \mathrm{C} \mathrm{NMR}\left(100 \mathrm{MHz}, \mathrm{CDCl}_{3}\right): 21.3\left(\mathrm{CH}_{3}\right)$, $55.5\left(\mathrm{OCH}_{3}\right), 103.7$ (Ar. CH), 115.7 (Ar. CH), 118.5 (Ar. CH), 120.3 (Ar. CH), 122.1 (Ar. CH), 123.5 (Ar. CH), 126.6 (ar. CH), 127.1 (Ar. CH), 129.6 (Ar. CH), 130.6 (Ar. CH), 131.6 (Ar. CH), 136.7 (Ar. CH), 139.1 (Ar. CH), 143.8 (Ar. CH), 144.9 (Ar. CH), 145.1 (Ar. CH), 155.0 (Ar. CH), 157.8 (Ar. CH), ESIMS: $[\mathrm{M}+\mathrm{H}]^{+} \mathrm{m} / \mathrm{z}$ : 341; Chemical Formula: $\mathrm{C}_{23} \mathrm{H}_{20} \mathrm{~N}_{2} \mathrm{O}$.

3. 2-(2-(4-Chlorophenyl)-6-Methoxyquinolin-4-Y1)Aniline (4c): The crude substance purified by gravity column chromatography (ethyl acetate/n-hexane 1:2).

Brown solid substance, yield 0.26 g (74\%), m.p. 174$176{ }^{\circ} \mathrm{C}, \mathrm{R}_{\mathrm{f}}: 0.70\left(\mathrm{SiO}_{2}\right.$, ethyl acetate/n-hexane 1:2). FTIR V: $3382\left(\mathrm{NH}_{2}\right), 3035,2809,2859($ Aromatic $=\mathrm{CH}), 1900,1615$, $1515(\mathrm{C}=\mathrm{C}$ ), 1082, 815,600 (mono-substituted aromatic ring $\mathrm{C}-\mathrm{Cl}$ ), ${ }^{1} \mathrm{H}$ NMR (400 MHz, $\left.\mathrm{CHCl}_{3}-d\right): \delta$ (ppm): 3.80(s, 3H, $\left.\mathrm{CH}_{3}\right), 6.87(\mathrm{~d}, 1 \mathrm{H}, \mathrm{Ar} .=\mathrm{CH}, J: 5.2 \mathrm{~Hz}), 6.96-6.1(\mathrm{~m}, 2 \mathrm{H}$, Ar. $=\mathrm{CH}), 7.21-7.19(\mathrm{~m}, 1 \mathrm{H}, \mathrm{Ar} .=\mathrm{CH}), 8.13-8.08(\mathrm{~m}, 3 \mathrm{H}, \mathrm{Ar} .=$ $\mathrm{CH}), 7.33-7.30(\mathrm{~m}, 1 \mathrm{H}, \mathrm{Ar} .=\mathrm{CH}), 7.41-7.39(\mathrm{~m}, 1 \mathrm{H}, \mathrm{Ar}$. $=\mathrm{CH}), 7.47(\mathrm{~d}, 2 \mathrm{H}, \mathrm{Ar} .=\mathrm{CH}, J: 6 \mathrm{~Hz}, 2 \mathrm{H}), 7.80(\mathrm{~s}, 1 \mathrm{H}, \mathrm{Ar}$. $=\mathrm{CH}),{ }^{13} \mathrm{C} \mathrm{NMR}\left(400 \mathrm{MHZ}, \mathrm{CDCl}_{3}\right): 55.5\left(\mathrm{OCH}_{3}\right), 103.7(\mathrm{Ar}$. CH), 113.6 (Ar. CH), 113.7 (Ar. CH), 118.5 (Ar. CH), 120.06 (Ar. CH), 122.46 (Ar. CH), 126.8 (Ar. CH), 128.5 (Ar. CH), 128.9 (Ar. CH), 129.7 (Ar. CH), 130.5 (Ar. CH), 131.6 (Ar. CH), 133.2 (Ar. CH), 135.2 (Ar. CH), 137.9 (Ar. CH), 143.7 (Ar. CH), 145.4 (Ar. CH), 158.1 (Ar. CH), ESI-MS: $[\mathrm{M}+\mathrm{H}]^{+}$ $\mathrm{m} / \mathrm{z} 361$; Chemical Formula: $\mathrm{C}_{22} \mathrm{H}_{17} \mathrm{ClN}_{2} \mathrm{O}$.

4. 2-(2-(4-Bromophenyl)-6-Methoxyquinolin-4-Y1)Aniline (4d): The crude substance purified by gravity column chromatography (ethyl acetate/n-hexane 1:2).

Brown solid substance, yield 0.30 g (75\%) m.p. 108$110^{\circ} \mathrm{C}, \mathrm{R}_{\mathrm{f}}: 0.70\left(\mathrm{SiO}_{2}\right.$, ethyl acetate/n-hexane 1:2). FTIR V: $3435\left(\mathrm{NH}_{2}\right), 3318,3205,3063,2916($ aromatic $=\mathrm{C}-\mathrm{H}), 2354$, 1917, 1626, 1583 (C=C), 1492, 1305, 1091, 1014, 823 (mono-substituted aromatic ring $\mathrm{CH}$ ) $\mathrm{cm}^{-1},{ }^{1} \mathrm{H} \mathrm{NMR}$ (400 MHz, $\left.\mathrm{CHCl}_{3}-d\right): \delta(\mathrm{ppm}): 3.81\left(\mathrm{~s}, 1 \mathrm{H}, \mathrm{OCH}_{3}\right), 6.99$ 6.88(m, 3H, Ar. -CH), 7.35-7.19(m, 2H, Ar. =CH), 7.517.41(m, 3H, Ar. $=\mathrm{CH}), 7.74(\mathrm{~s}, 1 \mathrm{H}, \mathrm{Ar} .=\mathrm{CH}), 8.14(\mathrm{~d}, 2 \mathrm{H}$, Ar. $=\mathrm{CH}, J: 8.4 \mathrm{~Hz}), 8.37(\mathrm{~s}, 1 \mathrm{H}$, Ar. $=\mathrm{CH}),{ }^{13} \mathrm{C} \mathrm{NMR}$ $\left(100 \mathrm{MHz}, \mathrm{CHCl}_{3}-d\right) \delta$ (ppm): $55.3\left(\mathrm{OCH}_{3}\right), 103.61$ (Ar. $\mathrm{CH}$ ), 115.7 (Ar. CH), 118.6 (Ar. CH), 120.0 (Ar. CH), 122.5 (Ar. CH), 123.3 (Ar. CH), 123.6 (Ar. CH), 126.9 (Ar. CH), 128.8 (Ar. CH), 129.7 (Ar. CH), 130.5 (Ar. CH), 131.6 (Ar. CH), 131.9 (Ar. CH), 143.7 (Ar. CH), 145.5 (ar. $\mathrm{CH}$ ), 144.8 (Ar. CH), ESI-MS: $[\mathrm{M}+\mathrm{H}]^{+} \mathrm{m} / \mathrm{z}$ : 405; Chemical Formula: $\mathrm{C}_{22} \mathrm{H}_{17} \mathrm{BrN}_{2} \mathrm{O}$.

5. 2-(6-Chloro-2-(Thiophen-2-Y1)Quinolin-4-Y1)Aniline (4e): The crude substance purified by gravity column chromatography (ethyl acetate/n-hexane 1:2). 
Table 1 Optimization of reaction conditions for the synthesis of 2,4-disubstituted quinoline derivative $(\mathbf{4 a})^{\mathrm{a}}$

\begin{tabular}{|c|c|c|c|c|c|c|}
\hline Entry & Catalyst & Mol (\%) of catalyst & Solvent & Reaction condition & Time (h) & Yield $^{\mathrm{b}}(\%)$ \\
\hline 1. & $\mathrm{InCl}_{3}$ & 5 & Toluene & Reflux & 24 & 65 \\
\hline 2. & $\mathrm{InCl}_{3}$ & 10 & Toluene & Reflux & 24 & 70 \\
\hline 3. & $\mathrm{InCl}_{3}$ & 15 & Toluene & Reflux & 24 & 70 \\
\hline 4. & $\mathrm{InCl}_{3}$ & 10 & $\mathrm{CH}_{3} \mathrm{CN}$ & Reflux & 24 & 55 \\
\hline 5. & $\mathrm{InCl}_{3}$ & 10 & DCM & Reflux & 24 & 50 \\
\hline 6. & $\mathrm{InCl}_{3}$ & 10 & $\mathrm{EtOH}$ & Reflux & 24 & 45 \\
\hline 7. & $\mathrm{InCl}_{3}$ & 10 & THF & Reflux & 24 & 40 \\
\hline 8. & $\mathrm{InCl}_{3}$ & 10 & $\mathrm{H}_{2} \mathrm{O}$ & Reflux & 48 & NR \\
\hline 9. & $\mathrm{InCl}_{3}$ & 10 & No solvent & $100{ }^{\circ} \mathrm{C}$ & 48 & NR \\
\hline 10. & $\mathrm{InCl}_{3}$ & 10 & $\mathrm{CH}_{3} \mathrm{NO}_{2}$ & Reflux & 24 & 50 \\
\hline 11. & $\mathrm{InBr}_{3}$ & 10 & Toluene & Reflux & 24 & 50 \\
\hline 12. & $\mathrm{AlCl}_{3}$ & 10 & Toluene & Reflux & 24 & 42 \\
\hline 13. & $\mathrm{FeCl}_{3}$ & 10 & Toluene & Reflux & 24 & 34 \\
\hline 14. & $\mathrm{CuCl}_{2}$ & 10 & Toluene & Reflux & 24 & 45 \\
\hline 15. & $\mathrm{Cu}(\mathrm{OTf})_{2}$ & 10 & Toluene & Reflux & 24 & Multi-spots \\
\hline 16. & CAN & 10 & Toluene & Reflux & 24 & 48 \\
\hline 17. & $\mathrm{I}_{2}$ & 10 & Toluene & Reflux & 24 & 45 \\
\hline 18. & TFA & 10 & Toluene & Reflux & 24 & 30 \\
\hline 19. & $p$-TsOH & 10 & Toluene & Reflux & 24 & 35 \\
\hline 20. & $\mathrm{BF}_{3} \mathrm{OEt}_{3}$ & 10 & Toluene & Reflux & 24 & 38 \\
\hline
\end{tabular}

${ }^{\text {a }}$ All the reactions were performed with aniline $\mathbf{1 a}(1.0 \mathrm{mmol})$, benzaldehyde $\mathbf{2 a}(1.0 \mathrm{mmol})$ and 2-ethynylaniline $3(1.0 \mathrm{mmol}){ }^{\mathrm{b}}$ Isolated yields NR- No reaction.
Brown solid substance, yield 0.24 g (72\%) m.p. 145$147^{\circ} \mathrm{C}, \mathrm{R}_{\mathrm{f}}: 0.70\left(\mathrm{SiO}_{2}\right.$, ethyl acetate/n-hexane 1:2). FTIR V: $3489\left(\mathrm{NH}_{2}\right), 3020,2939,2874($ aromatic $=\mathrm{C}-\mathrm{H}), 1963,1654$, $1530(\mathrm{C}=\mathrm{C}$ ), 1083, 906,708 (mono-substituted $\mathrm{C}$ aromatic ring $\mathrm{C}-\mathrm{Cl}) \mathrm{cm}^{-1} ;{ }^{1} \mathrm{H}$ NMR $\left(400 \mathrm{MHz}, \mathrm{CHCl}_{3}-d\right) \delta(\mathrm{ppm})$ : 6.51(s, $1 \mathrm{H}$, Ar. $=\mathrm{CH}) ; 7.03(\mathrm{t}, 1 \mathrm{H}, J: 4.8 \mathrm{~Hz}), 7.46-7.19(\mathrm{~m}$, $5 \mathrm{H}, \mathrm{Ar} .=\mathrm{CH}), 7.59(\mathrm{~s}, 1 \mathrm{H}, \mathrm{Ar} .=\mathrm{CH}), 7.62(\mathrm{~d}, 1 \mathrm{~h}, J: 7.2 \mathrm{~Hz})$, $7.78(\mathrm{~d}, 1 \mathrm{H}, J: 8.4 \mathrm{~Hz}), 7.98$ (d, $1 \mathrm{H}, J: 8.4 \mathrm{~Hz}),{ }^{13} \mathrm{C} \mathrm{NMR}$ (100 MHz, $\left.\mathrm{CHCl}_{3}-\mathrm{H}\right) \delta$ (ppm): 98.9 (Ar. CH), 119.4 (Ar. $\mathrm{CH}$ ), 123.7 (Ar. $\mathrm{CH}$ ), 125.2 (Ar. $\mathrm{CH}$ ), 127.9 (Ar. $\mathrm{CH}$ ), 128.2 (Ar. CH), 129.5 (Ar. CH), 129.9 (Ar. CH), 129.9 (Ar. $\mathrm{CH}), 130.1$ (Ar. CH), 138.6 (Ar. CH), 145.6 (Ar. CH), 147.4 (Ar. CH), 125.5 (Ar. CH), 152.9 (Ar. CH), MS: $[\mathrm{M}+\mathrm{H}]^{+} \mathrm{m} / \mathrm{z}$ 337. Chemical Formula: $\mathrm{C}_{19} \mathrm{H}_{13} \mathrm{ClN}_{2} \mathrm{~S}$.

6. 2-(6-Chloro-2-(4-Methoxyphenyl)Quinolin-4-Yl)Aniline (4f): The crude substance purified by gravity column chromatography (ethyl acetate/n-hexane 1:2).

Brown solid substance, yield $0.26 \mathrm{~g}$ (74\%) m.p. 138$140{ }^{\circ} \mathrm{C}, \mathrm{R}_{\mathrm{f}}: 0.70\left(\mathrm{SiO}_{2}\right.$, ethyl acetate/n-hexane 1:2). FTIR V: $3484\left(\mathrm{NH}_{2}\right), 3023,2932($ aromatic $=\mathrm{C}-\mathrm{H}), 2865,1960,1657$, $1596(\mathrm{C}=\mathrm{C}), 1068,907,760$ (mono-substituted aromatic ring $\mathrm{C}-\mathrm{H}) \mathrm{cm}^{-1} ;{ }^{1} \mathrm{H} \mathrm{NMR}(400 \mathrm{MHz}, \mathrm{CHCl} 3-d): \delta(\mathrm{ppm}): 3.55(\mathrm{~s}$, $\left.2 \mathrm{H}, \mathrm{NH}_{2}\right), 3.86\left(\mathrm{~s}, 3 \mathrm{H}, \mathrm{OCH}_{3}\right), 6.94-6.85(\mathrm{~m}, 3 \mathrm{H}, \mathrm{Ar} .=\mathrm{CH})$, $7.15(\mathrm{~d}, 1 \mathrm{H}, \mathrm{Ar} .=\mathrm{CH}, J: 7.2 \mathrm{~Hz}), 7.33-7.25(\mathrm{~m}, 2 \mathrm{H}, \mathrm{Ar} .=\mathrm{CH})$, $7.63(\mathrm{~d}, 2 \mathrm{H}, \mathrm{Ar} .=\mathrm{CH}, J: 10 \mathrm{~Hz}), 7.84(\mathrm{~s}, 1 \mathrm{H}, \mathrm{Ar} .=\mathrm{CH}), 8.12(\mathrm{t}$,
$3 \mathrm{H}, \mathrm{Ar} .=\mathrm{CH}, J: 4.8 \mathrm{~Hz}) ;{ }^{13} \mathrm{C} \mathrm{NMR}\left(100 \mathrm{MHz}, \mathrm{CHCl}_{3}-\mathrm{H}\right) \delta$ (ppm): 55.4( $\left(\mathrm{OCH}_{3}\right), 114.3$ (Ar. $\left.\mathrm{CH}\right), 115.8$ (Ar. $\left.\mathrm{CH}\right), 118.6$ (Ar. CH), 120.4 (Ar. CH), 122.6 (Ar. $\mathrm{CH}), 124.6$ (Ar.CH), 126.2 (Ar.CH), 128.9(Ar.CH), 130.0 (Ar.CH), 130.6(Ar.CH), 130.7 (Ar.CH), 132.0 (Ar.CH), 143.7 (Ar.CH), 145.8 (Ar.CH), 147.3 (Ar.CH), 157.1 (Ar.CH), 161.1 (Ar.CH); ESI-MS: $[\mathrm{M}+\mathrm{H}]^{+} \mathrm{m} / \mathrm{z}$ 361. Chemical Formula: $\mathrm{C}_{22} \mathrm{H}_{17} \mathrm{ClN}_{2} \mathrm{O}$.

7. 2-(6-Methyl-2-(p-Tolyl)Quinolin-4-Yl)Aniline (4 g): The crude substance purified by gravity column chromatography (ethyl acetate/n-hexane 1:2).

Brown solid substance, yield 0.23 g (72\%) m.p. 145$147^{\circ} \mathrm{C}, \mathrm{R}_{\mathrm{f}}: 0.70(\mathrm{SiO} 2$, ethyl acetate/n-hexane 1:2). FTIR V: $3474\left(\mathrm{NH}_{2}\right), 3013,2899($ aromatic $=\mathrm{C}-\mathrm{H}), 2854,1990$, 1657, $1526(\mathrm{C}=\mathrm{C}$ ), 1078, 879,731 (mono-substituted aromatic ring $\mathrm{C}-\mathrm{H}) \mathrm{cm}^{-1} ;{ }^{1} \mathrm{H}$ NMR $\left(400 \mathrm{MHz}, \mathrm{CHCl}_{3}-d\right)$ : $\delta(\mathrm{ppm})$ : $2.38\left(\mathrm{~s}, 3 \mathrm{H}, \mathrm{CH}_{3}\right), 2.49\left(\mathrm{~s}, 3 \mathrm{H}, \mathrm{CH}_{3}\right), 3.49\left(\mathrm{~s}, 2 \mathrm{H}, \mathrm{NH}_{2}\right)$, $6.95(\mathrm{~d}, 1 \mathrm{H}$, Ar. $=\mathrm{CH}, J: 8.8 \mathrm{~Hz}), 6.87-6.63(\mathrm{~m}, 3 \mathrm{H}, \mathrm{Ar}$. $=\mathrm{CH}), 7.11(\mathrm{~d}, 1 \mathrm{H}, J: 7.6 \mathrm{~Hz}), 7.26-7.18(\mathrm{~m}, 1 \mathrm{H}, \mathrm{Ar} .=\mathrm{CH})$, $7.33(\mathrm{~s}, 1 \mathrm{H}, \mathrm{Ar} .=\mathrm{CH}), 7.47(\mathrm{~d}, 1 \mathrm{H}, \mathrm{Ar} .=\mathrm{CH}, J: 8.4 \mathrm{~Hz}), 7.71(\mathrm{~s}$, $1 \mathrm{H}, \mathrm{Ar} .=\mathrm{CH}), 8.07-8.01(\mathrm{dd}, 3 \mathrm{H}, J: 8.4,8.8),{ }^{13} \mathrm{C} \mathrm{NMR}$ $\left(100 \mathrm{MHz}, \mathrm{CHCl}_{3}-\mathrm{H}\right) \delta(\mathrm{ppm}): 21.3\left(\mathrm{CH}_{3}\right), 21.8\left(\mathrm{CH}_{3}\right)$, 115.6 (Ar. CH), 118.4(Ar. CH), 120.1 (Ar. CH), 123.7 (Ar. $\mathrm{CH}), 124.4$ (Ar. CH), 125.7 (Ar. CH), 127.3(Ar. CH), 129.5 (Ar. CH), 129.8(Ar. CH), 130.6 (Ar. $\mathrm{CH}), 132.1$ (Ar. CH), 
Table 2 Synthesis of various 2,4-disubstituted quinoline derivatives using Povarov reaction. ${ }^{\text {a }}$

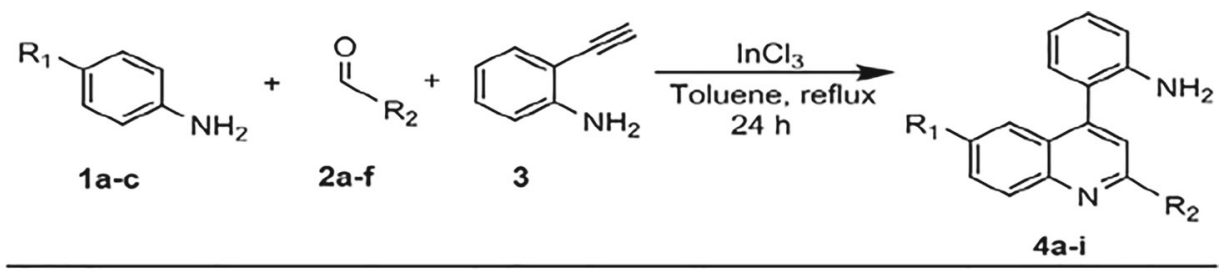<smiles>Nc1ccccc1-c1cc(-c2ccccc2)nc2ccccc12</smiles>

$4 a, 70 \%$<smiles>COc1ccc2nc(-c3ccc(Br)cc3)cc(-c3ccccc3N)c2c1</smiles>

$4 d, 75 \%$<smiles>Cc1ccc(-c2cc(-c3ccccc3N)c3cc(C)ccc3n2)cc1</smiles>

$4 \mathrm{~g}, 72 \%$<smiles>COc1ccc2nc(-c3ccc(C)cc3)cc(-c3ccccc3N)c2c1</smiles>

$4 b, 73 \%$<smiles>Nc1ccccc1-c1cc(-c2cccs2)nc2ccc(Cl)cc12</smiles>

$4 e, 72 \%$<smiles>Cc1ccc2nc(-c3ccc(Cl)cc3)cc(-c3ccccc3N)c2c1</smiles>

4h, $73 \%$<smiles>COc1ccc2nc(-c3ccc(Cl)cc3)cc(-c3ccccc3N)c2c1</smiles>

4c. $74 \%$<smiles>COc1ccc(-c2cc(-c3ccccc3N)c3cc(Cl)ccc3n2)cc1</smiles><smiles>Cc1ccc2nc(-c3ccc([N+](=O)[O-])cc3)cc(-c3ccccc3N)c2c1</smiles>

$4 i, 75 \%$

${ }^{\text {a }}$ Reactions condition: Aromatic amines $(1.0 \mathrm{mmol})$, aromatic aldehydes $(1.0 \mathrm{mmol})$, and 2-ethynylaniline $(1.0 \mathrm{mmol})$ in the presence of $10 \mathrm{~mol} \% \mathrm{of}$ $\mathrm{InCl}_{3}$ in $5 \mathrm{~mL}$ of toluene under reflux conditions.

136.4 (Ar. CH), 143.9 (Ar. CH), 145.8 (Ar. CH), 147.4 (Ar. $\mathrm{CH})$; ESI-MS: $[\mathrm{M}+\mathrm{H}]^{+} \mathrm{m} / \mathrm{z}$ 325. Chemical Formula: $\mathrm{C}_{23} \mathrm{H}_{20} \mathrm{~N}_{2}$.

8. 2-(2-(4-Chlorophenyl)-6-Methylquinolin-4-Yl)Aniline $(4 \mathrm{H})$ : The crude substance purified by gravity column chromatography (ethyl acetate/n-hexane 1:2).

Brown solid substance, yield $0.25 \mathrm{~g}(73 \%)$ m.p. $96-98^{\circ} \mathrm{C}$, $\mathrm{R}_{\mathrm{f}}: 0.70\left(\mathrm{SiO}_{2}\right.$, ethyl acetate/n-hexane 1:2). FTIR V: 3474 $\left(\mathrm{NH}_{2}\right), 3005,2945($ aromatic $=\mathrm{C}-\mathrm{H}), 2867,1970,1661$, $1546(\mathrm{C}=\mathrm{C}), 1078,900,872$ (mono-substituted aromatic ring $\mathrm{C}-\mathrm{H}) \mathrm{cm}^{-1} ;{ }^{1} \mathrm{H}$ NMR $\left(400 \mathrm{MHz}, \mathrm{CHCl}_{3}-d\right): \delta(\mathrm{ppm}): 2.45(\mathrm{~s}$, $\left.3 \mathrm{H}, \mathrm{CH}_{3}\right), 3.52\left(\mathrm{~s}, 2 \mathrm{H}, \mathrm{NH}_{2}\right), 6.94-6.85(\mathrm{~m}, 2 \mathrm{H}, \mathrm{Ar} .=\mathrm{CH})$, 7.18-7.16(m, 1H, Ar. =CH), 7.25(s, 1H, Ar. =CH), 7.34$7.30(\mathrm{~m}, 2 \mathrm{H}, \mathrm{Ar} .=\mathrm{CH}), 7.47(\mathrm{t}, 1 \mathrm{H}, \mathrm{Ar} .=\mathrm{CH}, J: 8.8 \mathrm{~Hz})$, $7.58-7.55(\mathrm{~m}, 1 \mathrm{H}, \mathrm{Ar} .=\mathrm{CH}), 7.8(\mathrm{~s}, 1 \mathrm{H}, \mathrm{Ar} .=\mathrm{CH}), 8.11(\mathrm{~d}$, $3 \mathrm{H}$, Ar. $=\mathrm{CH}, J: 8.8 \mathrm{~Hz}) ;{ }^{13} \mathrm{C} \mathrm{NMR}\left(100 \mathrm{MHz} \mathrm{CDCl}_{3}\right): 20.8$
$\left(\mathrm{CH}_{3}\right), 115.7$ (Ar. $\left.\mathrm{CH}\right), 118.5$ (Ar. $\left.\mathrm{CH}\right), 119.8$ (Ar. $\left.\mathrm{CH}\right), 123.4$ (Ar. CH), 124.4(Ar. CH), 125.8 (Ar. $\mathrm{CH}), 128.7$ (Ar. CH), 129.0 (Ar. CH), 129.6 (Ar. CH), 129.9 (Ar. CH), 130.6 (Ar. $\mathrm{CH}), 132.3$ (Ar. $\mathrm{CH}$ ), 135.4 (Ar. CH), 136.9 (Ar. CH), 137.9 (Ar. CH), 143.8 (Ar. CH), 146.2 (Ar. CH), 147.3 (Ar. CH), 155.1 (Ar. CH); ESI-MS: $[\mathrm{M}+\mathrm{H}]^{+} \mathrm{m} / \mathrm{z}$ 345. Chemical Formula: $\mathrm{C}_{22} \mathrm{H}_{17} \mathrm{ClN}_{2}$.

9. 2-(6-Methyl-2-(4-Nitrophenyl)Quinolin-4-Yl)Aniline (4i): The crude substance purified by gravity column chromatography (ethyl acetate/n-hexane 1:2).

Brown solid substance, yield $0.26 \mathrm{~g}(75 \%)$ m.p. 167$169{ }^{\circ} \mathrm{C}, \mathrm{R}_{\mathrm{f}}: 0.70\left(\mathrm{SiO}_{2}\right.$, ethyl acetate/n-hexane $\left.1: 2\right)$. FTIR V: $3494\left(\mathrm{NH}_{2}\right), 3028,2938($ aromatic $=\mathrm{C}-\mathrm{H})$, 2877, 1950, 1650, $1536(\mathrm{C}=\mathrm{C}), 1068,877,721$ (monosubstituted aromatic ring $\mathrm{C}-\mathrm{H}) \mathrm{cm}^{-1} ;{ }^{1} \mathrm{H}$ NMR $(400 \mathrm{MHz}$, $\left.\mathrm{CHCl}_{3}-d\right): \delta(\mathrm{ppm}): 2.50\left(\mathrm{~s}, 3 \mathrm{H}, \mathrm{CH}_{3}\right), 3.57\left(\mathrm{~s}, 2 \mathrm{H}, \mathrm{NH}_{2}\right.$ ), 
(a)<smiles>CCOC(=O)c1cccc(N)c1</smiles>

$1 e$<smiles>COc1ccc(C=O)cc1</smiles>

$2 f$<smiles>C#Cc1ccccc1N</smiles>

$\underset{\text { conditions }}{\stackrel{\text { Standard }}{\longrightarrow}}$<smiles>CCOC(=O)c1cccc(/N=C/c2ccc(OC)cc2)c1</smiles>

(b)<smiles>C#Cc1ccccc1NC(C)=O</smiles>

Scheme 2 Controlled experiment to established the effect of amino group in this Povarov reaction.

6.98-6.90(m, 2H, Ar. =CH), 7.20(d, 2H, J: 7.6), 7.26(s, 1H, Ar. $=\mathrm{CH}), 7.37(\mathrm{t}, 1 \mathrm{H}$, Ar. $=\mathrm{CH}, J: 8.0 \mathrm{~Hz}), 7.45(\mathrm{~s}, 1 \mathrm{H}, \mathrm{Ar}$. $=\mathrm{CH}), 7.72-7.62(\mathrm{~m}, 1 \mathrm{H}, \mathrm{Ar} .=\mathrm{CH}), 7.89(\mathrm{~s}, 1 \mathrm{H}, \mathrm{Ar} .=\mathrm{CH})$, 8.16 (d, 1 h, J: $8.4 \mathrm{~Hz}), 8.55$ (d, 1H, J: 7.6 Hz), 9.07(s, 1H, Ar. $=\mathrm{CH}) ;{ }^{13} \mathrm{C}$ NMR $\left(100 \mathrm{MHz}, \mathrm{CDCl}_{3}\right): 21.8\left(\mathrm{CH}_{3}\right), 115.7$ (Ar. $\mathrm{CH}), 118.5$ (Ar. CH), 119.8 (Ar. CH), 123.4 (Ar. CH), 124.4 (Ar. CH), 125.8 (Ar. CH), 128.7 (Ar. CH), 129.0 (Ar. CH), 129.6 (Ar. CH), 129.9 (Ar. CH), 130.6 (Ar. CH), 132.3 (Ar. $\mathrm{CH}), 135.4$ (Ar. $\mathrm{CH}), 137.0$ (Ar. CH), 138.0 (Ar. $\mathrm{CH}), 143.8$
(Ar. CH), 146.2 (Ar. CH), 147.3 (Ar. CH), 155.1 (Ar. CH); ESI-MS: $[\mathrm{M}+\mathrm{H}]^{+} \mathrm{m} / \mathrm{z}$ 356. Chemical Formula: $\mathrm{C}_{22} \mathrm{H}_{17} \mathrm{~N}_{3} \mathrm{O}_{2}$.

10. Ethyl (E)-3-((4-Methoxybenzylidene)Amino)Benzoate (2j'): The crude substance purified by gravity column chromatography (ethyl acetate/n-hexane 1:2).

Brownish solid substance, yield 0.26 g (92\%) m.p. 58$62{ }^{\circ} \mathrm{C}, \mathrm{R}_{\mathrm{f}}: 0.70\left(\mathrm{SiO}_{2}\right.$, ethyl acetate/n-hexane $\left.1: 2\right)$. FTIR V:

Scheme 3 Proposed mechanism for the synthesis of Quinoline 4a

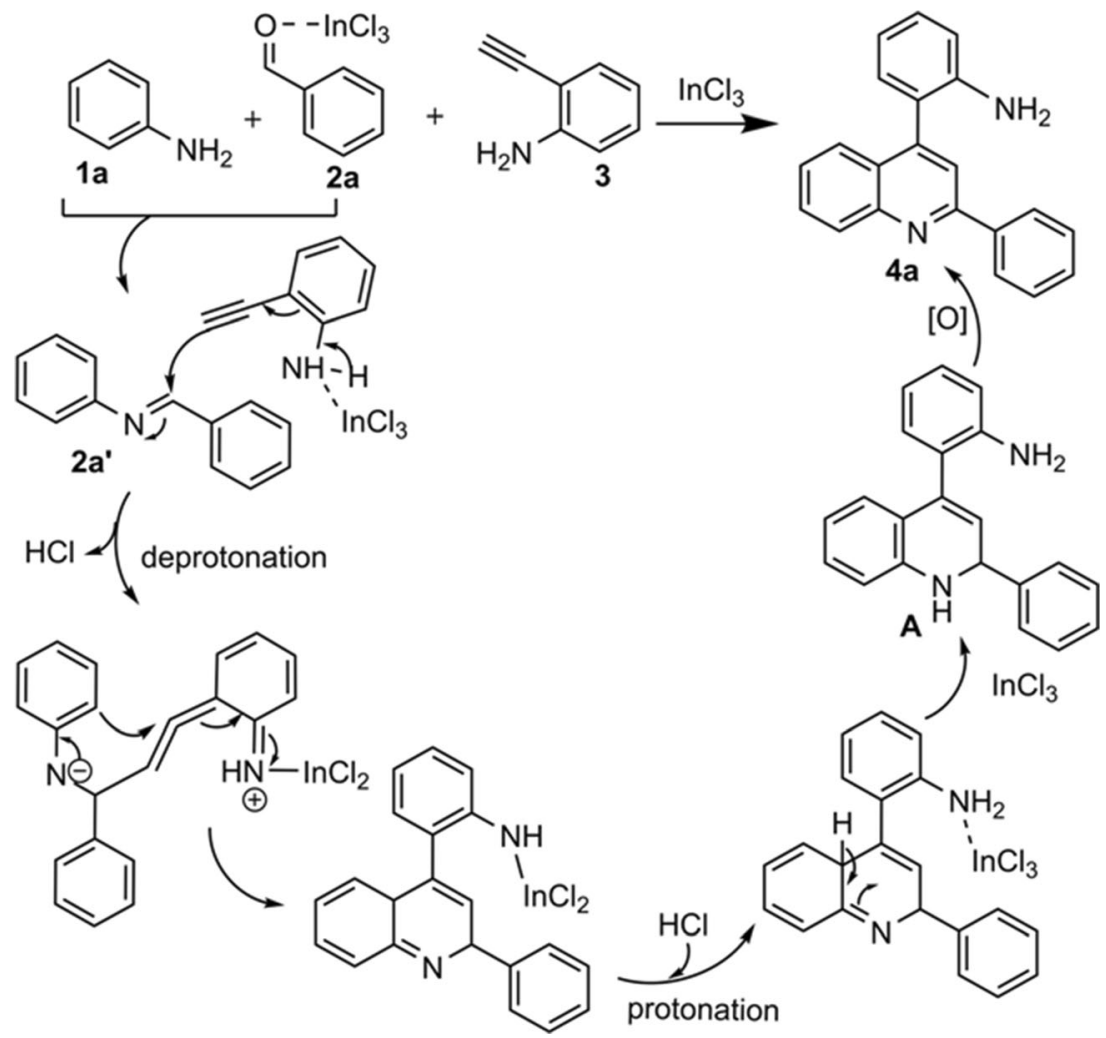


Table 3 Maximum absorption wavelength $\left(\lambda_{\max }\right)$ for quinoline derivatives

\begin{tabular}{|c|c|c|c|c|c|c|c|}
\hline \multirow[t]{2}{*}{ Sl. No. } & \multirow[t]{2}{*}{ Compounds } & \multicolumn{2}{|l|}{ Chloroform } & \multicolumn{2}{|l|}{ Acetonitrile } & \multicolumn{2}{|l|}{ Methanol } \\
\hline & & Peaks (nm) & $\lambda_{\max }(\mathrm{nm})$ & Peaks (nm) & $\lambda_{\max }(\mathrm{nm})$ & Peaks (nm) & $\lambda_{\max }(\mathbf{n m})$ \\
\hline 1. & $4 a$ & 249,319 & 249 & 250,318 & 250 & 281,319 & 281 \\
\hline 2. & $4 b$ & 250,387 & 250 & 264,383 & 264 & 263,385 & 263 \\
\hline 3. & $4 c$ & 264,333 & 333 & 260,319 & 318 & 268,322 & 322 \\
\hline 4. & $4 d$ & 262,372 & 262 & 268,351 & 268 & 264,348 & 264 \\
\hline 5. & $4 e$ & 267,337 & 267 & 268,332 & 268 & 267,332 & 267 \\
\hline 6. & $4 f$ & 265,348 & 265 & 271,342 & 271 & 270,344 & 270 \\
\hline 7. & $4 \mathrm{~g}$ & 262,347 & 262 & 279,342 & 279 & 280,345 & 280 \\
\hline 8. & $4 \mathrm{~h}$ & 260,380 & 260 & 260,384 & 260 & 260,379 & 260 \\
\hline 9. & $4 i$ & 260,343 & 260 & 261,326 & 261 & 260,324 & 260 \\
\hline
\end{tabular}

3067 (aromatic $=$ C-H), 2987, 1705, 1612 $(\mathrm{C}=\mathrm{N}), 1413,1365$, 870 (monosubstituted aromatic ring $\mathrm{C}-\mathrm{H}$ ) $\mathrm{cm}^{-1} ;{ }^{1} \mathrm{H} \mathrm{NMR}$ (400 MHz, $\mathrm{CHCl}_{3}-d$ ) $\delta(\mathrm{ppm}): 1.37$ (t, 3H, $-\mathrm{CH}_{3}, J: 6.8 \mathrm{~Hz}$ ), 3.82(s, 3H, $\left.\mathrm{OCH}_{3}\right), 4.37-4.31(\mathrm{dd}, 2 \mathrm{H}, \mathrm{J}$ : 7.2, 7.2 Hz), 6.79$6.77(\mathrm{~m}, 2 \mathrm{H}, \mathrm{Ar} .=\mathrm{CH}), 6.88(\mathrm{~d}, 1 \mathrm{H}, \mathrm{J}: 8.8 \mathrm{~Hz}), 7.19(\mathrm{~s}, 1 \mathrm{H}$, Ar. $=\mathrm{CH}), 7.22(\mathrm{~d}, 1 \mathrm{H}, \mathrm{J}: 4.0 \mathrm{~Hz}), 7.26$ (t, 1H, J: $6.4 \mathrm{~Hz}), 7.33$ (d, 1H, J: $5.6 \mathrm{~Hz}$ ), 7.38 (d, 1H, J: $7.6 \mathrm{~Hz}), 8.52$ (s, 1H, Ar. = $\mathrm{CH}$, 13C NMR-APT (100 MHz, CHCl3-d) $\delta$ (ppm): 14.3 $\left(\mathrm{CH}_{3}\right), 55.3\left(\mathrm{OCH}_{3}\right), 60.9\left(-\mathrm{CH}_{2}\right), 117.1$ (Ar. $\left.\mathrm{CH}\right), 114.1$ (Ar. CH), 113.5 (Ar. CH), 114.3 (Ar. CH), 118.6 (Ar. CH), 128.0 (Ar. CH), 129.2 (Ar. CH), 130.9 (Ar. CH), 131.3 (Ar. $\mathrm{CH}), 148.1$ (Ar. CH), 158.9 (Ar. CH), $167.0(\mathrm{C}=\mathrm{N})$; ESI-MS: $[\mathrm{M}+\mathrm{H}]^{+} \mathrm{m} / \mathrm{z}$ 284. Chemical Formula: $\mathrm{C}_{17} \mathrm{H}_{17} \mathrm{NO}_{3}$.

\section{Results and Discussion}

Initially, a mixture of aniline $\mathbf{1 a}(1.0 \mathrm{mmol})$, benzaldehyde $\mathbf{2 a}$ (1.0 mmol) and 2-ethynylaniline $3(1.0 \mathrm{mmol})$ with $\mathrm{InCl}_{3}$ ( $5 \mathrm{~mol} \%$ ) were refluxed for $24 \mathrm{~h}$ in $5 \mathrm{~mL}$ of toluene, $(E)$ $N$-(2-ethynylphenyl)-1-phenylmethanimine and traces of unreacted aniline were isolated instead of our target compound quinolines. Then, we modified the reaction in a

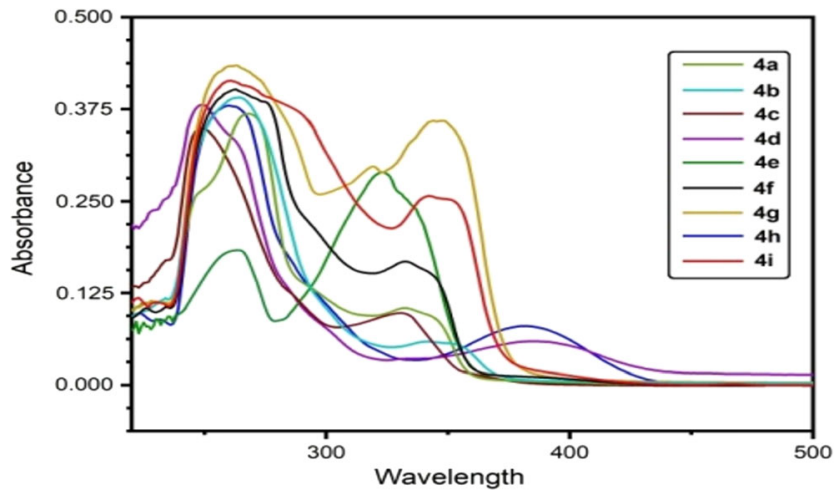

Fig. 2 UV-Vis absorption of quinoline derivatives $(\mathbf{4 a - i})$ in $\mathrm{CH}_{3} \mathrm{Cl}$ stepwise controlled method so that aniline $\mathbf{1 a}(1.0 \mathrm{mmol})$ and benzaldehyde $\mathbf{2 a}(1.0 \mathrm{mmol})$ were allowed to react in refluxing toluene in presence of $5 \mathrm{~mol} \%$ of $\mathrm{InCl}_{3}$ for $1 \mathrm{~h}$ showing the characteristic formation of imine (as indicated by TLC) and 2-ethynylaniline $3(1.0 \mathrm{mmol})$ was added to reaction pot and refluxed the system for another $23 \mathrm{~h}$. The product 2-(2-phenylquinolin-4-yl)aniline 4 a was obtained in $65 \%$ yield (Table 1, entry 1 ), the structure of $\mathbf{4 a}$ was deduced from its elemental analysis and spectral data $\left({ }^{1} \mathrm{H}\right.$ and ${ }^{13} \mathrm{C}$ NMR, IR). To our delight, the desired product 4a was obtained in $70 \%$ yield when we increased the catalytic loading to $10 \mathrm{~mol} \%$ (entry 2). However, no increase in yield was observed on further increasing the catalyst beyond $10 \mathrm{~mol} \%$ (entry 3). Inspired by this result, we next screened the effect of solvent on the reaction by using $10 \mathrm{~mol} \%$ of $\mathrm{InCl}_{3}$ as standard catalyst loading. For scrutinizing the suitable solvent system, similar reactions (entries 4-8) were conducted in various solvent systems such as $\mathrm{CH}_{3} \mathrm{CN}$, DCM, EtOH, THF and $\mathrm{CH}_{3} \mathrm{NO}_{2}$ under reflux conditions. It was noted that the shortest reaction time and the best yield were obtained in toluene (entry 2) under reflux condition. Interestingly, the same reaction did not proceed and provide low yield when it was carried out in water and solvent free condition even after prolonging the reaction duration (Table 1, entries 9-10).

To examine the efficacy of $\mathrm{InCl}_{3}$ extensive comparative studies with several catalytic systems have been investigated. Thus, several reactions were scrutinized in the presence of catalysts like $\mathrm{InBr}_{3}, \mathrm{AlCl}_{3}, \mathrm{FeCl}_{3}, \mathrm{Cu}(\mathrm{OTf})_{2}, \mathrm{CuCl}_{2}, \mathrm{CAN}$, $\mathrm{I}_{2}$, TFA, $p$-TsOH and $\mathrm{BF}_{3} \mathrm{OEt}_{3}$, respectively (Table 1 , entries 11-20). All the tested acids gaive lower yields than that of $\mathrm{InCl}_{3}$, however, in the case of $\mathrm{Cu}(\mathrm{OTf})_{2}$, a mixture of compounds are observed. Lewis acids showed better activity as compared to those of Bronsted acids and amongst the tested Lewis acids $\mathrm{InCl}_{3}$ was the best catalyst in this Povarov reaction (Table 1). Hence, the optimized reaction condition for the formation of $\mathbf{4 a}$ was established with $10 \mathrm{~mol} \%$ of $\mathrm{InCl}_{3}$ in toluene under refluxing for $24 \mathrm{~h}$ using aniline $1 \mathrm{a}(1.0 \mathrm{mmol})$, 
benzaldehyde $2 \mathrm{a}(1.0 \mathrm{mmol})$ and 2-ethynylaniline 3 (1.0 mmol) (entry 2).

With this optimized reaction condition in our hand, its substrate scope and generality was examined (Table 2). Amines with electron-donating substituents such as methoxy and methyl react with aldehydes having different substituents such as methoxy, methyl, chloro, bromo and nitro in presence of 2ethynylaniline to give moderate yields $(72-75 \%)$. Again, $p$ chloroaniline react with $p$-methoxybenzaldehyde or thienyl-2carbaldehyde along with 2-ethynylaniline to give the corresponding quinolines in comparable yields with other products. However, the best yields were obtained when amines having the electron-donating groups coupled with aldehydes having electron-withdrawing substituents such as in $\mathbf{4 d}$ and $\mathbf{4 i}$ (75\% yield). Thus, a small library of highly functionalized quinolines with potentials of exhibiting strong luminescence properties under UV lamp was established. We were curious to find out the role of amino group which is ortho to enyne moiety.

To understand the role of amino group, an experiment for ethyl 3-aminobenzoate $1 \mathrm{e}(1.0 \mathrm{mmol}), p$-methylbenzaldehyde $2 \mathbf{f}(1.0 \mathrm{mmol})$ and 2-ethynylaniline $\mathbf{3}(1.0 \mathrm{mmol})$ were subjected to establish optimized reaction condition (Table 1, entry 2). The intermediate Schiff base $\mathbf{2 j}$ ' was obtained instead of expected quinoline, probably due to the reduction in the electron density on ortho to the ester carbon as well as steric hindrance between the amino group and bulky acetate. Thus, less chances for the formation of quinoline.

In another experiment, the $-\mathrm{NH}_{2}$ of ethynylaniline was acylated with acetic anhydride and allowed to react with isolated Schiff base 2c' using the established protocol. Again, the reaction mixture gives multi-spot as observed by TLC, indicating that the amino group must be involved in the cycloaddition reaction between the Schiff base and 2-ethynylaniline as shown in Scheme 2. Taking into consideration of the above two observations, we propose the plausible reaction mechanism of this Povarov reaction to get quinoline via oxidation. Here, the Schiff base 2a' undergo [4+2] Povarov cycloaddition reaction with 2-ethynylaniline initiated from amino group. The cyclized dihydroquinoline $\mathbf{A}$ is obtained with the departure of the $\mathrm{InCl}_{3}$ catalyst and restoration of the catalytic cycle. Then dihydroquinoline A undergoes a spontaneous oxidation to give the final quinoline $4 \mathbf{a}$ (Scheme 3).

\section{Photophysical Properties}

In this study, we examine the absorption, emission, life time and fluorescence quantum yields of the quinolines. The photophysical characteristics were investigated in $\mathrm{CH}_{3} \mathrm{CN}$, $\mathrm{CHCl}_{3}$ and $\mathrm{CH}_{3} \mathrm{OH}$ solutions. The data of $\mathrm{UV}-\mathrm{Vis}$ absorption

Table 4 Photophysical data obtained from fluorescence emission of quinoline derivatives

\begin{tabular}{|c|c|c|c|c|c|c|c|}
\hline Sl. No. & Compounds & $\lambda_{\mathrm{em}}$ & $\Delta \lambda \mathbf{s t}$ & Parameters & $\begin{array}{l}\text { Average } \\
\text { Life-Time }\left(\tau_{\mathrm{av}}\right)\end{array}$ & $\begin{array}{l}\text { Quantum } \\
\text { Yield } \Phi f x\end{array}$ & $\begin{array}{l}\text { CIE } \\
\text { Coordinate }\end{array}$ \\
\hline 1. & $4 a$ & 454 & 90 & $\begin{array}{l}\text { (a) } \tau_{1}=1.366 \\
\text { (b) } \tau_{2}=5.494 \\
\text { (c) } \chi^{2}=0.999\end{array}$ & $2.745 \mathrm{~ns}$ & 0.01 & $\begin{array}{l}X=0.214 \\
Y=0.200\end{array}$ \\
\hline 2. & $4 b$ & 438 & 100 & $\begin{array}{l}\text { (a) } \tau_{1}=1.681 \\
\text { (b) } \tau_{2}=4.733 \\
\text { (c) } \chi^{2}=0.999\end{array}$ & $1.805 \mathrm{~ns}$ & 0.01 & $\begin{array}{l}X=0.160 \\
Y=0.095\end{array}$ \\
\hline 3. & $4 c$ & 490 & 94 & $\begin{array}{l}\text { (a) } \tau_{1}=2.704 \\
\text { (b) } \tau_{2}=10.742 \\
\text { (c) } \chi^{2}=0.999\end{array}$ & $3.313 \mathrm{~ns}$ & 0.09 & $\begin{array}{l}X=0.259 \\
Y=0.510\end{array}$ \\
\hline 4. & $4 d$ & 491 & 95 & $\begin{array}{l}\text { (a) } \tau_{1}=3.700 \\
\text { (b) } \tau_{2}=7.510 \\
\text { (c) } \chi^{2}=0.999\end{array}$ & $4.177 \mathrm{~ns}$ & 0.20 & $\begin{array}{l}X=0.266 \\
Y=0.550\end{array}$ \\
\hline 5. & $4 e$ & 492 & 95 & $\begin{array}{l}\text { (a) } \tau_{1}=4.307 \\
\text { (b) } \tau_{2}=7.409 \\
\text { (c) } \chi^{2}=0.999\end{array}$ & $7.266 \mathrm{~ns}$ & 0.09 & $\begin{array}{l}X=0.323 \\
Y=0.042\end{array}$ \\
\hline 6. & $4 f$ & 488 & 108 & $\begin{array}{l}\text { (a) } \tau_{1}=2.304 \\
\text { (b) } \tau_{2}=12.175 \\
\text { (c) } \chi^{2}=0.999\end{array}$ & $3.435 \mathrm{~ns}$ & 0.04 & $\begin{array}{l}X=0.223 \\
Y=0.604\end{array}$ \\
\hline 7. & $4 \mathrm{~g}$ & 480 & 99 & $\begin{array}{l}\text { (a) } \tau_{1}=1.861 \\
\text { (b) } \tau_{2}=8.341 \\
\text { (c) } \chi^{2}=0.999\end{array}$ & $5.598 \mathrm{~ns}$ & 0.02 & $\begin{array}{l}X=0.125 \\
Y=0.112\end{array}$ \\
\hline 8. & $4 \mathrm{~h}$ & 496 & 77 & $\begin{array}{l}\text { (a) } \tau_{1}=2.488 \\
\text { (b) } \tau_{2}=8.322 \\
\text { (c) } \chi^{2}=0.999\end{array}$ & $6.204 \mathrm{~ns}$ & 0.78 & $\begin{array}{l}X=0.245 \\
Y=0.525\end{array}$ \\
\hline 9. & $4 i$ & 472 & 92 & $\begin{array}{l}\text { (a) } \tau_{1}=2.314 \\
\text { (b) } \tau_{2}=10.852 \\
\text { (c) } \chi^{2}=0.999\end{array}$ & $3.210 \mathrm{~ns}$ & 0.16 & $\begin{array}{l}X=0.165 \\
Y=0.181\end{array}$ \\
\hline
\end{tabular}



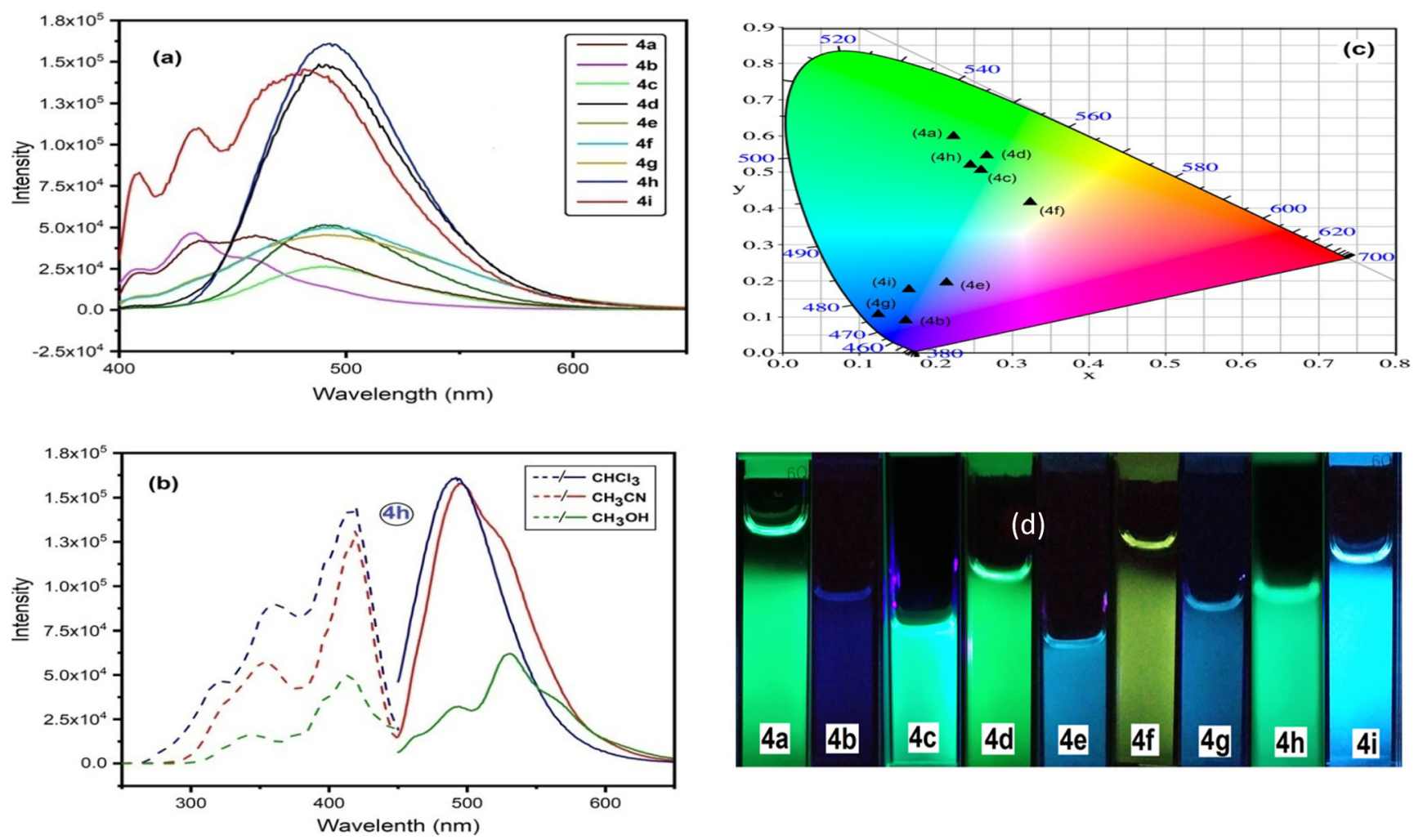

Fig. 3 a Fluorescence emission of quinoline derivatives $(\mathbf{4 a - i})$ in $\mathrm{CHCl}_{3}$ (b), Emission (solid line) and absorption (dotted line) spectra of compound $4 \mathbf{h}$ in each solvent $\left(\mathrm{CHCl}_{3}, \mathrm{CH}_{3} \mathrm{CN}, \mathrm{CH}_{3} \mathrm{OH}\right)(\mathbf{c})$,

are summarized in Table 3 in $10^{-3}$ mol. $\mathrm{L}^{-1}$ in $\mathrm{CH}_{3} \mathrm{CN}, \mathrm{CHCl}_{3}$ and $\mathrm{CH}_{3} \mathrm{OH}$ solutions.

The nature of the substituents were examined taking $4 \mathbf{a}$ as reference compound, it was found that all the compounds exhibit red shifts. The highest shift occurred in 2-position thienyl substituted $\mathbf{4 e}$, as expected $(18 \mathrm{~nm})$. The absorption spectra of the 2,4-disubstituted quinoline derivatives in $\mathrm{CHCl}_{3}$ are characterized by strong absorption peaks centred at 249 $267 \mathrm{~nm}$ and $319-387 \mathrm{~nm}$ probably due to $\pi-\pi^{*}$ and $\mathrm{n}-\pi^{*}$

Photograph taken under UV light $(365 \mathrm{~nm})$ in $\mathrm{CHCl}_{3}$ solution. d Chromaticity diagram showing the CIE coordinates of the compounds 4a-i

transitions. Bands located at $319-387 \mathrm{~nm}$ range can be assigned to the possible intermolecular charge transfer transition (ICT) [35]. On changing the polarity to polar solvents like $\mathrm{CH}_{3} \mathrm{CN}$ and $\mathrm{CH}_{3} \mathrm{OH}$, most of the compounds experience red shift and blue shift respectively for $\pi-\pi^{*}$ and $n-\pi^{*}$ peaks. The $\lambda_{\max }$ changes by $31 \mathrm{~nm}$ from acetonitrile to methanol for $\mathbf{4 a}$ (Table 3). It can be attributed to possible protonation of quinolines in methanol. However, the compound $4 \mathbf{c}$ showed blue shift in both the transitions in $\mathrm{CH}_{3} \mathrm{CN}$ from $\mathrm{CHCl}_{3}$. In all the

Fig. 4 Fluorescence lifetime decays of quinolines $(\mathbf{4 a - i})$

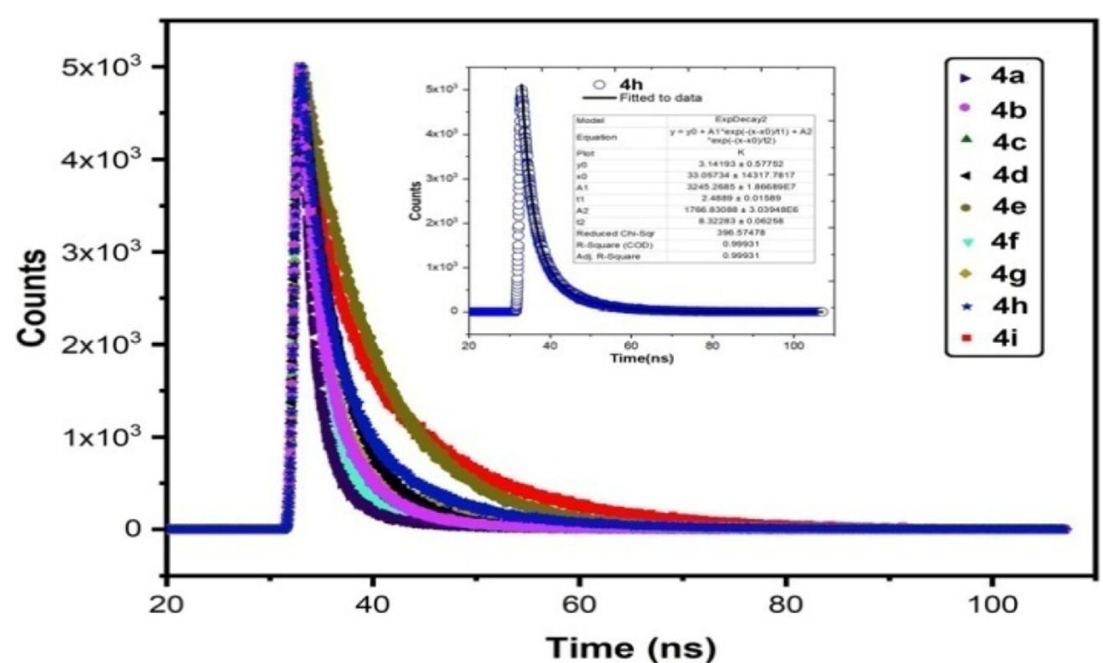


compounds the change in $\lambda_{\max }$ due to substituent's nature seems to be less pronounced. This can be attributed due to the strong influence of amino group at $o$-position of the aryl substituent at position 4, no significant alteration was observed by changing the substituents on the phenyl ring in position 2 as well as at position 6 of quinolines (Fig. 2). All the compounds showed $\lambda_{\max }$ in $\pi-\pi^{*}$ transition however, compound $\mathbf{4 c}$ is the exception with $\lambda_{\max } 323 \mathrm{~nm}$ (n- $\pi^{*}$ transition) having methoxy and chloro as substituents in all the solvents (absorption spectra in $\mathrm{CH}_{3} \mathrm{CN}$ and $\mathrm{CH}_{3} \mathrm{OH}$ are enclosed in SI). The absorption spectra of chloroform solutions $\left(10^{-3}\right.$ mol. $\left.\mathrm{L}^{-1}\right)$ of 2,4-disubstituted quinoline derivatives are depicted in Fig. 2.

The fluorescence and excitation spectra were measured with a Horiba Fluoromax 4 Spectrophotometer. All measurements were done repeatedly and reproducible results were obtained. All the solutions were excited at around 338$419 \mathrm{~nm}$ with an excitation and emission slit width of $2 \mathrm{~nm}$. As in absorption, the solvent polarity affects the fluorescence properties of quinolines. The Stokes shift data, given by the difference between the maximum peak of absorption and emission spectra which is estimated from the intersection of the absorption and emission spectra, is observed in Table 4 for $\mathrm{CHCl}_{3}$ solution. Most of the compounds showed a similar pattern in the shift around 472-496 nm whereas 4a $(454 \mathrm{~nm})$ and $\mathbf{4 b}(438 \mathrm{~nm})$ as expected show the shift at lower wavelength. The larger Stoke's shift values of 77-108 can be attributed to the ICT transitions and electron-substituent properties that exist in these compounds. They showed green to blue emission under UV-lamp (365 nm) except 4 f with yellowish colour (Fig. 3c) and Commission Internationale de L'Eclairage (CIE) colour coordinates of the compounds are summarized in Table 4 (also in Fig. 3d).

When the difference in fluorescence intensity was analyzed in $\mathrm{CHCl}_{3}$, compounds $\mathbf{4 d}, \mathbf{4} \mathbf{h}$ and $\mathbf{4 i}$ presented greater intensity, with $\mathbf{4} \mathbf{h}$ being the highest one (Fig. $3 \mathrm{a}$ ). On changing the polarity of the solvents, the shifts pattern are drastically affected with low intensity observing in polar-protic solvent $\mathrm{CH}_{3} \mathrm{OH}(\mathbf{S 3})$ than polar aprotic solvent $\mathrm{CH}_{3} \mathrm{CN}(\mathbf{S 4})$. Whereas, compounds $\mathbf{4 d}, \mathbf{4} \mathbf{h}$ and $\mathbf{4 i}$ remain with greater intensity than other in both the polar solvents like in $\mathrm{CHCl}_{3}$. The intensity of peaks decreased significantly owing to the hydrogen-bonding interaction between $\mathrm{NH}_{2}$ group of $4 \mathbf{a}-\mathbf{i}$ and protic-solvent in $\mathrm{CH}_{3} \mathrm{OH}$ along with polarity effect, but no such hydrogen-bonding with $\mathrm{CH}_{3} \mathrm{CN}$ having only polar effect where $\mathrm{CHCl}_{3}$ has none of the effect (Fig. 3d).

The fluorescence quantum yields $(\varphi)$ of all the compounds in $\mathrm{CHCl}_{3}$ solution are also summarized in Table 4 . The higher $\varphi$ values were obtained in three compounds i.e., $0.78,0.20$, and 0.16 for $\mathbf{4} \mathbf{h}, \mathbf{4 d}$, and $\mathbf{4 i}$ respectively. And, for other compounds $\varphi$ values ranges between $0.01-0.09$ in $\mathrm{CHCl}_{3}$ solution. The $\mathrm{CH}_{3} \mathrm{CN}$ solution exhibits lower values due to its polarity $(0.75,0.18$, and 0.15 for $\mathbf{4} \mathbf{h}, \mathbf{4 d}$, and $\mathbf{4 i})$. The lower $\varphi$ values $(0.54,0.05$, and 0.01 for $\mathbf{4} \mathbf{h}, \mathbf{4 d}$, and $\mathbf{4 i})$ in $\mathrm{CH}_{3} \mathrm{OH}$ solution is due to its higher polarity.

\section{Average Lifetime}

Average lifetime ( $\mathrm{av}$ ) measurements for all the newly synthesized compounds have been carried out. It was found that 4e (7.266 ns) has highest lifetime followed by $\mathbf{4} \mathbf{h}(6.20 \mathrm{~ns})$, $\mathbf{4} \mathbf{g}(5.60 \mathrm{~ns}), \mathbf{4 d}(\mathbf{4 . 1 8} \mathrm{ns}), \mathbf{4 f}(3.44 \mathrm{~ns}), \mathbf{4 c}(3.313 \mathrm{~ns}), \mathbf{4 i}$ (3.21 ns), $4 \mathbf{a}(2.75 \mathrm{~ns})$ and $\mathbf{4 b}(1.81 \mathrm{~ns})$ (Fig. 4). The average lifetime signifies that species with higher tav is likely to persist for longer period in the excited state. Thus, compounds $\mathbf{4 e}$, $\mathbf{4 ~ h , ~} \mathbf{4} \mathbf{g}$ and $\mathbf{4 d}$ can be explored for potential candidates in fluorescence imaging.

\section{Conclusion}

In summary, $\mathrm{InCl}_{3}$-catalyzed [4+2] cycloaddition reaction giving novel quinoline derivatives having an amino group at the 4-aryl substituent is presented. To the best of our knowledge, the use of 2-ethynylaniline in Povarov reaction is not reported till now except our work. All the newly synthesized quinolines gave intense green/blue fluorescence with large Stokes shifts. Some of the compounds also have good quantum yield as well as reasonable life time. Studies expanding this novel modular approach to enhance molecular diversity with better yields and more detailed photophysical investigations are currently underway for their photonic applications.

Supplementary Information The online version contains supplementary material available at https://doi.org/10.1007/s10895-020-02647-3.

Acknowledgments Financial support from the DST, CSIR and DBT (BT/PR25414/NER/95/ 1183/2017), Government of India, are gratefully appreciated.

\section{Compliance with Ethical Standards}

Conflict of Interest The authors declare that they have no conflict of interest.Supplementary Information The online version contains supplementary material available at https://doi.org/10.1007/s10895-02002647-3.

\section{References}

1. Michael JP (2007) Quinoline, quinazoline and acridone alkaloids. Nat Prod Rep 24:223-246

2. Prajapati SM, Patel KD, Vekariya RH, Panchal SN, Patel HD (2014) Recent advances in the synthesis of quinolines: a review. RSC Adv 4:24463-24476 
3. Shang XF, Morris NSL, Liu YQ, Guo X, Xu XS, Goto M, Li JC, Yang GZ, Lee KH (2018) Biologically active quinoline and quinazoline alkaloids part I. Med Res Rev 38:775-828

4. Candeias NR, Branco LC, Gois PMP, Afonso CAM, Trindade AF (2009) More sustainable approaches for the synthesis of n-based heterocycles. Chem Rev 109:2703-2802

5. Nqoro X, Tobeka N, Aderibigbe BA (2017) Quinoline-based hybrid compounds with antimalarial activity. Molecules 22:2268

6. Guan LP, Jin QH, Wang SF, Li FN, Quan ZS (2008) Synthesis and evaluation on anticonvulsant and antidepressant activities of 5-alkoxy-tetrazolo[1,5-a]quinazolines. Arch Pharm 341:774-779

7. Ibrahim DA, El Ella DAA, El-Motvvally AM, Aly RM (2015) 3Formylchromones as diverse building blocks in heterocycles synthesis. Eur J Med Chem 102:115-131

8. Pierre F, O'Brien SE, Haddach M, Bourbon P, Schwaebe MK, Stefan E, Darjania L, Stansfield R, Ho C, Siddiqui JA, Streiner N, Rice WG, Anderes K, Ryckman RM (2011) A subnanomolar fluorescent probe for protein kinase CK2 interaction studies. Bioorg Med Chem Lett 21:1687-1691

9. Gautret P, Lagier JC, Parola P, Hoang VT, Meddeb L, Mailhe M, Doudier B, Courjon J, Giordanengo V, Vieira VE, Dupont HT, Honoré S, Colson P, Chabrière E, La Scola B, Rolain JM, Brouqui P, Raoult D (2020) Hydroxychloroquine and azithromycin as a treatment of COVID-19: results of an open-label non-randomized clinical trial. Int J Antimicrob Agents 56:105949

10. Wall ME, Wani MC, Cook CE, Keith PH, McPhail AT, Sim GA (1966) Plant antitumor agents. I. the isolation and structure of camptothecin, a novel alkaloidal leukemia and tumor inhibitor from Camptotheca acuminate. J Am Chem Soc 88:3888-3890

11. Liu YQ, Li WQ, Morris NS, Qian K, Yang L, Zhu GX, Wu XB, Chen AL, Zhang SY, Nan X, Lee KH (2015) Design and synthesis of novel PEG-conjugated 20(S)-camptothecin sulfonylamidine derivatives with potent in vitro antitumor activity via cu-catalyzed three-component reaction. Med Res Rev 35:753-789

12. dos Santos GC, Servilha RO, de Oliveira EF, Lavarda FC, Ximenes VF, da Silva-Filho LC (2017) Theoretical-experimental photophysical investigations of the solvent effect on the properties of green- and blue-light-emitting quinoline derivatives. J Fluoresc 27:1709-1720

13. Pourfallah G, Lou X (2018) Novel 4-amino-2-methyl-8(trifluoromethyl)quinoline-based magnetic nanostructures for highly sensitive detection of zinc ions in aqueous solutions. Dyes Pigments 158:12-19

14. Grimsdale AC, Chan KL, Martin RE, Jokisz PG, d Holmes AB (2009) Synthesis of light-emitting conjugated polymers for applications in electroluminescent devices. Chem Rev 109:897-1091

15. Mikeska LA, Haller HL, Adams EQ (1920) Anodic oxidation pathways of substituted triphenylamines. II Quantitative studies of benzidine formation J Am Chem Soc 42:2392-2394

16. Dumouchel S, Mongin F, Trécourt F, Quéguiner G (2003) Tributyl magnesium ate complex-mediated bromine-magnesium exchange of bromoquinolines: a convenient access to functionalized quinolines. Tetrahedron Lett 44:2033-2035

17. Goel A, Kumar V, Singh SP, Sharma A, Prakash S, Singh C, Anand RS Non-aggregating solvatochromic bipolar benzo $[f]$ quinolines and benzo $[a]$ acridines for organic electronics. J Mater Chem 22: $14880-14888$

18. Zhang X, Kale DM, Jenekhe SA (2002) Electroluminescence of multicomponent conjugated polymers. 2. Photophysics and enhancement of electroluminescence from blends of polyquinolines. Macromolecules 35:382-393
19. Povarov LS (1967) A silver-catalyzed domino route toward 1,2dihydroquinoline derivatives from simple anilines and alkynes. Russian Chem Rev 36:656-670

20. Kouznetsov VV (2009) Recent synthetic developments in a powerful imino Diels-Alder reaction (Povarov reaction): application to the synthesis of $N$-polyheterocycles and related alkaloids. Tetrahedron 65:2721-2750

21. Jiang KM, Kang JA, Jin Y, Lin J (2018) Synthesis of substituted 4hydroxyalkyl-quinoline derivatives by a three-component reaction using $\mathrm{CuCl} / \mathrm{AuCl}$ as sequential catalysts. Org Chem Front 5:434 441

22. Xiao F, Chen Y, Liu Y, Wang J (2008) Sequential catalytic process: synthesis of quinoline derivatives by $\mathrm{AuCl}_{3} / \mathrm{CuBr}$-catalyzed threecomponent reaction of aldehydes, amines, and alkynes. Tetrahedron 64:2755-2761

23. Pericherla K, Kumar A, Jha A (2008) Povarov-reductive amination cascade to access 6-aminoquinolines and anthrazolines. Org Lett 15:4078-4081

24. Yao C, Qin B, Zhang H, Lu J, Wang D, Tu S (2012) One-pot solvent-free synthesis of quinolines by $\mathrm{C}-\mathrm{H}$ activation/ $\mathrm{C}-\mathrm{C}$ bond formation catalyzed by recyclable iron(III) triflate. RSC Adv 2: 3759-3764

25. Meyet CE, Larsen CH (2014) One-step catalytic synthesis of alkylsubstituted Quinolines. J Org Chem 79:9835-9841

26. Sarode PB, Bahekar SP, Chandak HS (2016) Zn(OTf $)_{2}$-mediated $\mathrm{C}-\mathrm{H}$ activation: an expeditious and solvent-free synthesis of aryl $/$ alkyl substituted quinolines. Tetrahedron Lett 57:5753-5756

27. Zhang X, Liu B, Shu X, Gao Y, Lv H, Zhu J (2012) Silver-mediated $\mathrm{C}-\mathrm{H}$ activation: oxidative coupling/cyclization of $\mathrm{N}$-arylimines and alkynes for the synthesis of quinolines. J Org Chem 77:501-510

28. Andrade AD, dos Santos GC, da Silva-Filho LC (2015) Facile synthesis and photophysical characterization of new quinoline dyes. J Heterocycl Chem 52:273-277

29. Yadav JS, Antony A, George J, Reddy BVS (2010) Multicomponent reactions using indium (III) salts. Curr Org Chem 14: 414-424

30. Mahato SK, Acharya C, Wellington KW, Bhattacharjee P, Jaisankar $\mathrm{P}$ (2020) $\mathrm{InCl}_{3}$ : a versatile catalyst for synthesizing a broad spectrum of heterocycles. ACS Omega 5:2503-2519

31. Li J, Li CJ (2001) Synthesis of tetrahydropyran derivatives via a novel indium trichloride mediated cross-cyclization between epoxides and homoallyl alcohols. Tetrahedron Lett 42:793-796

32. Singh TP, Bhattarcharya S, Singh OM (2013) Indium/TFAcatalyzed synthesis of tetracyclic $[6,5,5,6]$ indole ring, via a tandem cycloannulation of $\beta$-oxodithioester with tryptamine. Org Lett 15 : 1974-1977

33. Singh TP, Khan R, Noh YR, Lee SG, Singh OM (2014) Indium (III) chloride mediated michael addition of indoles to ketene S, Sacetals: synthesis of bis-and tris-indolylketones. Bull Kor Chem Soc 35:2950-2954

34. Singh TP, Devi TJ, Singh NP, Singh OM (2018) GFP chromophores from 1-phenylalanine: synthesis, photophysical and thermal properties. ChemistrySelect 3:6596-6600

35. Kulkarni AP, Wu PT, Kwon TW, Jenekhe SA (2005) Phenothiazine-phenylquinoline donor-acceptor molecules: effects of structural isomerism on charge transfer photophysics and electroluminescence. J Phys Chem B 109:19584-19594

Publisher's Note Springer Nature remains neutral with regard to jurisdictional claims in published maps and institutional affiliations. 